\title{
Red de Huertos Urbanos Comunitarios de Madrid. Prácticas y reflexiones colectivas
}

\author{
Belén Martínez Madrid, * Javier Garrido García, ** Pablo Llobera Serra*** y Aída \\ Rodríguez García****
}

Hábitat y Sociedad (ISSN 2173-125X), n. ${ }^{\circ}$ 6, noviembre de 2013, pp. 129-137.

\footnotetext{
Dpto. Medicina y Cirugía Animal, Facultad de Veterinaria, Universidad Complutense de Madrid (UCM).

** Universidad Complutense de Madrid. *** "Tablao de la compostura" (huerto comunitario del barrio de Las Tablas-Madrid) y de la ReHdMad!

**** Universidad Politécnica de Madrid

1 En el blog de la ReHdMad! (http:// redhuertosurbanosmadrid.wordpress. com), en un tono simpático a la vez que riguroso, podrá el lector interesado conocer con algún detalle muchas de estas experiencias, además de obtener información general sobre la red y sobre los huertos que la componen.

2 Las actividades, innovaciones y peculiaridades de cada huerto que participa en la red son numerosas, como no podía ser menos tratándose de un colectivo que aglutina huertos impulsados por asociaciones de vecinos, colegios, universidades, centros sociales y otros tipos de asociaciones formales e informales.
}

La Red de Huertos Urbanos Comunitarios de Madrid (ReHdMad!) nació en diciembre de 2010 para coordinar iniciativas de horticultura urbana comunitarias, gratuitas, abiertas y ecológicas. Sus objetivos apuntan a fomentar el intercambio de experiencias, reforzar los mecanismos de apoyo mutuo y los espacios formativos, facilitar el diálogo con la Administración Municipal y prestar orientación a entidades interesadas en iniciativas similares. Hoy, a los tres años de su nacimiento, el número de huertos miembros de la ReHdMad! ha pasado de media docena a más de treinta, y se ha puesto en marcha la negociación con el Ayuntamiento de la capital para proceder a la regularización del conjunto de huertos comunitarios. En definitiva, podemos afirmar que la ReHdMad! ha contribuido de muy distintas maneras al fortalecimiento de las iniciativas de agricultura urbana en la ciudad, convirtiéndose en un puntal fundamental del polifacético movimiento de los huertos urbanos comunitarios.

Las actividades y experiencias desarrolladas han sido muchas y diversas, constituyendo en su conjunto un proceso colectivo de aprendizaje muy intenso, que abarca dimensiones relacionadas, por ejemplo, con la agroecología, la educación ecológica, la ecología social, el uso de los espacios públicos, la autogestión ciudadana, las metodologías participativas, la puesta en práctica de valores marginados por el sistema, etc. ${ }^{1}$ En este breve texto daremos cuenta de algunas de ellas, acompañando su descripción con sintéticas reflexiones de los autores sobre el sentido y significado que las orientan. Por razones de espacio y de coherencia, nos centraremos únicamente en el trabajo en red y no abordaremos ninguna actividad realizada por cada huerto en el marco de su propia dinámica particular. ${ }^{2}$ Comenzaremos resumiendo algunos rasgos generales de organización y contextualización de la ReHdMad! y pasaremos después a describir y comentar varias experiencias de trabajo en red.

\section{Espacios de Encuentro: asambleas, encuentros, bicicletadas, comidas populares y talleres de reflexión}

La ReHdMad! se organiza y funciona mediante una asamblea mensual a la que asisten y participan miembros de los huertos que forman la Red. En ellas se ponen en común el estado, problemas, avances y dudas de los huertos, y se discuten muchos de los puntos que tienen que ver con las relaciones hacia el exterior. Estas asambleas tienen como caracterís- 


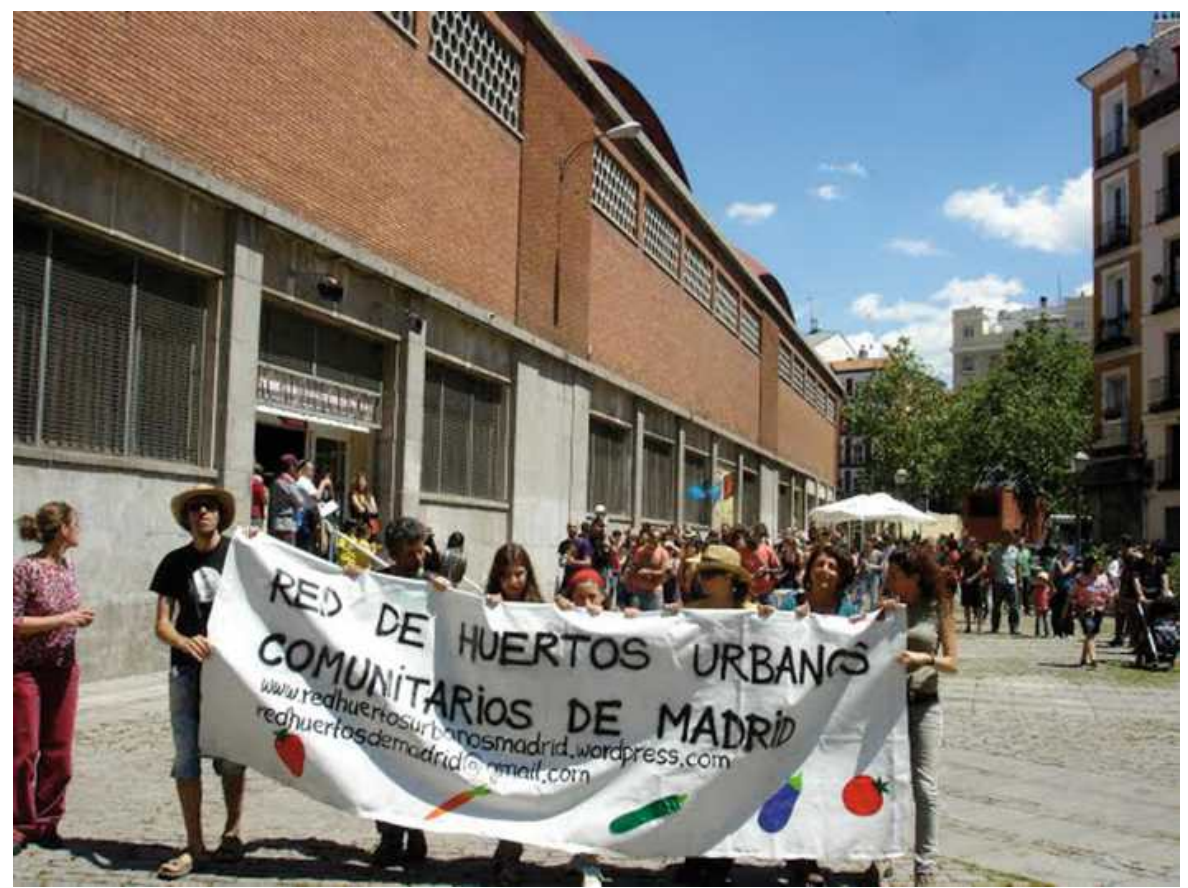

Figura 1. Paseo de la Red. Fuente: Elaboración propia. tica su rotación, celebrándose cada vez en un huerto, lo cual facilita el aprendizaje directo a los participantes, pues permite observar in situ las experiencias, soluciones y planteamientos que han elaborado otros hortelanos de la Red. También son momentos importantes de convivencia los Encuentros Anuales (2 hasta la fecha, mayo de 2012 y de 2013), planteados de forma festiva a lo largo de una jornada en la que se desarrollan talleres, se comparten conocimientos, se ponen en contacto hortelanos de distintos huertos y se da a conocer este movimiento social a un público más amplio. Finalmente, otras iniciativas puntuales que han ido surgiendo como espacios de encuentro son las Bicicletadas (mayo 2011, semana de lucha campesina 2013, Semana de la Ciencia 2012 y 2013) y las Comidas Populares.

Todos ellos son ejemplos de algunos valores importantes en la ReHdMad!: la creación de empatías y simpatías, de vivencias afectivas comunitarias, de acercamiento al espacio de los otros, de pensamiento y acción en los lugares de trabajo cotidianos. Se trata, en definitiva, de integrar trabajo y goce colectivo, razones y emociones, naturaleza y ciudad, en espacios concretos y significativos. En este sentido, cualquier fórmula de encuentro cobra especial relevancia, pues en primer lugar somos una red de personas, y es el hecho de conocerse y compartir lo que permite su evolución y desarrollo, adaptándose a los cambios internos y externos, dotando a esta iniciativa de una particular resiliencia. Sin embargo, no se trata sólo de encuentros personales, también hay reflexión sistematizada y continuada. Muestra de ello son los cuatro talleres que se han desarrollado hasta la fecha, en reuniones convocadas expresamente con el fin de pensarnos y reflexionar sobre la praxis de los huertos. Su análisis y resultados merecen un artículo aparte, baste aquí con indicar que han tratado temas como la organización, gestión y funcionamiento de los huertos; la socialización de los huertos en su contexto; la elaboración de propuestas de actuación; y la puesta en marcha o ejecución de acciones concretas para mejorar los procesos de los huertos comunitarios.

\section{Comunicación, difusión y relaciones con otros actores sociales}

Pero ni los huertos ni la RehdMad! adquieren plena entidad si no es dentro del entramado social al que pertenecen y en conexión con otros movimientos sociales. En este sentido, las interacciones con distintos movimientos y organizaciones con los que se comparten en general principios y estrategias, como la ISAm (Iniciativa por la Soberanía Alimentaria de Madrid), el 15M, centros sociales, DecreceMadrid, UniPosible o Ecologistas en Acción, por citar casos conocidos, son con- 
tinuas y generan cruciales avances, tanto en aspectos prácticos como ideológicos y de principios de la ReHdMad!

También las relaciones con el vecindario adquieren una importancia estratégica en la dinámica de los huertos, llevándose a cabo distintas iniciativas de interacción, como el apoyo a las fiestas de los barrios, la colaboración en eventos como las Jornadas Agroecológicas de Lavapiés, la Semana de Lucha Campesina y por la Soberanía Alimentaria, y actividades de centros escolares y de mayores próximos. De igual modo, la comunidad académica es otro de los actores con los que participamos activamente. Un buen número de estudiantes (incluidos miembros de la ReHdMad!) han realizado proyectos académicos en colaboración con nosotros, un trabajo simbiótico en el que los aportes de los huertos son devueltos a través de estudios que sistematizan nuestras experiencias, estructuras y funciones. Por último, seguir tejiendo alianzas (AMPAs, asociaciones vecinales, medios de comunicación, programas internacionales como Hábitat) e interrelaciones con otras redes, es una estrategia fundamental para la consolidación, legitimación y visibilidad de la ReHdMad! Tal es el caso concreto de uno de los recientes proyectos colectivos a los que nos hemos sumado: la Mesa de Proyectos Ciudadanos de Gestión de lo Público, "La Mesa Ciudadana”, un espacio de trabajo que investiga y promueve procesos de participación ciudadana en la gestión de lo público, la cual se reúne, generalmente, en Intermediae/MediaLab-Prado/Matadero.

En relación a la comunicación y difusión de la ReHdMad! hacia el exterior, ya en el I Encuentro contábamos con el Blog de la ReHdMad!, la exposición fotográfica itinerante "Vamos de Huertos", un Folleto informativo y el Mapa de Huertos Comunitarios de Madrid. ${ }^{3}$ El blog y el mapa se iniciaron gracias al desarrollo de un Trabajo Fin de Máster (Ventura, 2011). El Mapeo se desarroola como proceso de articulación con otros movimientos sociales de Madrid (DecreceMadrid, ISAm y ConsumaResponsabilidad), y se integra dentro de un proyecto más amplio, Mapunto, ${ }^{4}$ que pretende cartografiar las iniciativas sociales y de economía alternativa existentes en diferentes territorios del país.

Para finalizar este apartado, recordemos que en el área de comunicación se ha puesto especial énfasis en propiciar espacios donde sensibilizar, compartir debate y construir discurso sobre el fenómeno de la horticultura urbana comunitaria. Para ello se han organizado actividades como los Encuentros Anuales y se han aprovechado espacios emblemáticos de la ciudad, como La Casa Encendida (donde se celebró, por ejemplo, el ciclo de coloquios "Ruta 99: Dando vueltas a los huertos") o el Matadero-Intermediae (con el estreno del documental "De Madrid al suelo, la emergencia de la agricultura urbana" elaborado por el Centro de Medios Audiovisuales de la UNED). No menos importante ha sido la participación en foros de debate promovidos desde otras entida-
Figura 2. Fiesta en un huerto. Fuente: Elaboración propia.

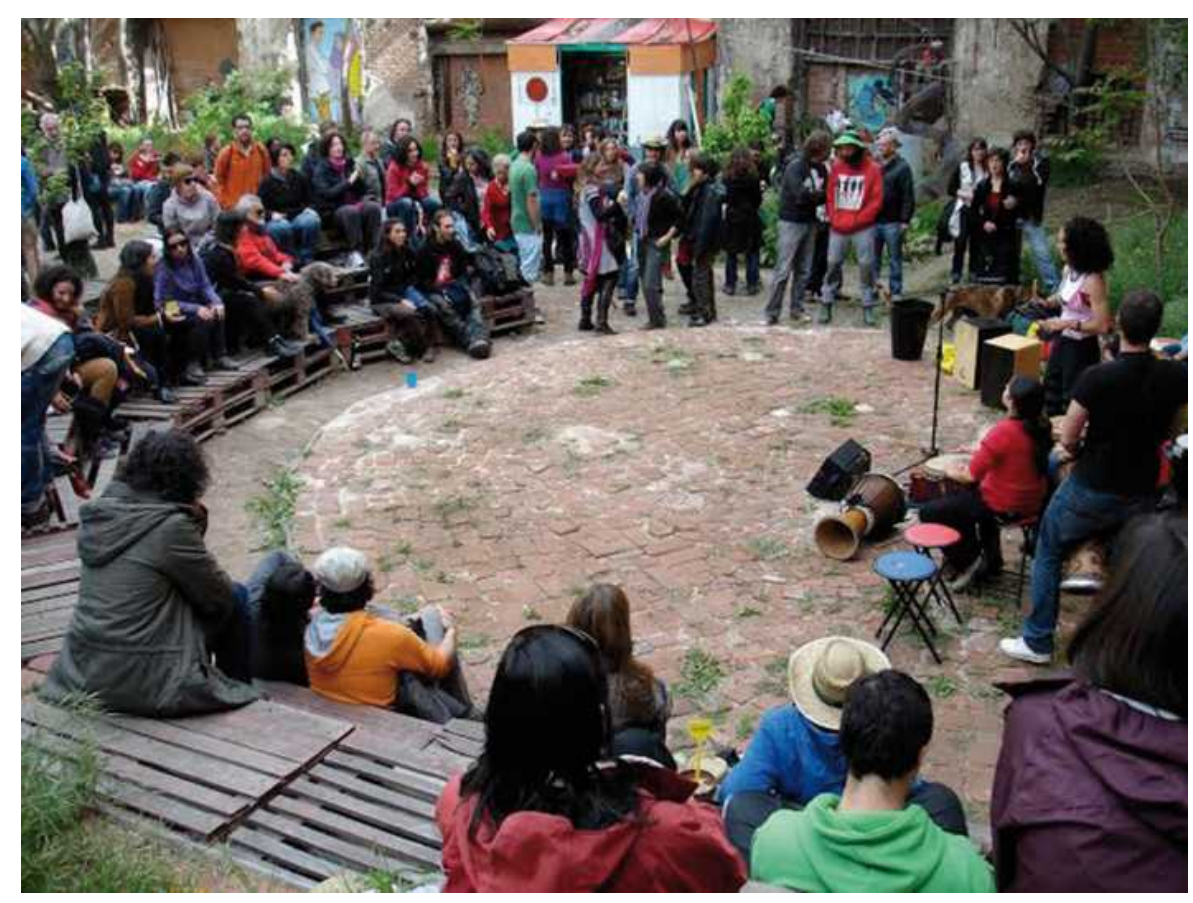




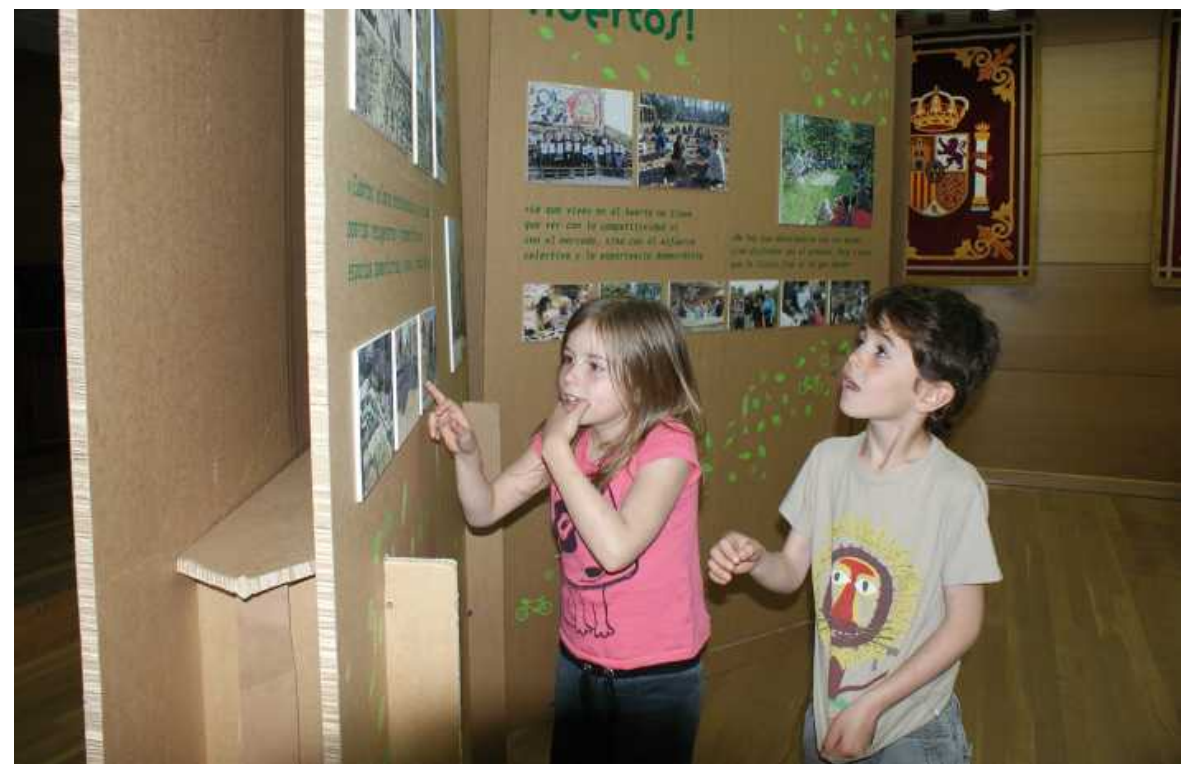

des, como el Aula de Agroecología de la Universidad Popular en Sierra Norte de Madrid (UniPoSiBLE) o el Congreso Nacional de Medioambiente (CONAMA). Y es bastante numerosa ya la aparición de la ReHdMad! o de alguno de los huertos en prensa, radio y televisión, ${ }^{5}$ así como la creación propia de materiales audiovisuales en diferentes huertos.

Cabría continuar exponiendo otros rasgos relacionados con la organización, identidad y marco de trabajo de la ReHdMad!, pero consideramos que en el marco de esta sección de la revisa son suficientes para dar al lector una idea de la Red

Figura 3. Niña y niño en la Exposición Fotográfica itinerante. Fuente: Elaboración propia. y para ubicar adecuadamente las experiencias de trabajo que pasamos a describir y comentar.

\section{Semillas y Semilleros: Semillero Comunitario}

En una lógica de organización y difusión del conocimiento y de la producción de carácter comunitario, absolutamente contrario al sentido privatizador del modelo de investigación, gestión y producción de semillas transgénica de las grandes compañías agrícolas, la ReHdMad! ha puesto en marcha varias experiencias de trabajo relacionadas con semillas. Así, en 2012, en el I Encuentro de la ReHdMad!, se puso en común la información sobre fuentes de obtención de semillas de variedades locales, tanto de forma gratuita a través de bancos de semillas institucionales (el INIA o el IMIDRA, por ejemplo) y de asociaciones agroecológicas (Ecosecha, La Troje o Zahoz, entre otras), como a partir de la compra en diferentes establecimientos. Del mismo modo, se compartió información sobre los espacios formales de intercambio de semillas (el coordinado por Ecosecha en el Matadero de Madrid) o informales (los que tienen lugar en las reuniones mensuales de la ReHdMad!). En esa misma línea de trabajo, cabe señalar la organización del $1^{\text {er }}$ Intercambio de Semillas y el Taller de Semillas, ambos celebrados en el marco de la Semana de Lucha Campesina en Madrid 2013, además de la organización en diferentes huertos de la Red de Talleres de Semillado para niños y mayores; de Obtención, Multiplicación y Conservación de Semillas; y de Elaboración de Bombas de Semillas. Y, él último y más reciente ejemplo, la distribución en ocho huertos de la red de la siembra experimental de semillas de algunas variedades de trigo procedentes de los antiguos silos de cereales de la dictadura franquista.

Siendo, pues, numerosos los ejemplos relacionados con éste campo de actuación y aprendizaje de la ReHdMad!, queremos destacar aquí el "Semillero Comunitario", experiencia que surge de la necesidad de compartir dos recursos, uno tangible, el invernadero preparado para la producción de plantel, ya que no todos los huertos de la Red disponen de esa infraestructura; y otro intangible, el conocimiento colectivo sobre la producción de plantel hortícola. La experiencia se inicia en 2012 en el invernadero de la HuertAula Comunitaria de Agroecología 
Cantarranas, de la UCM, y continúa en la campaña 2013, sumándose a la misma huertos que no participaron en la primera. En cada una de las campañas se organiza una reunión de planificación, en la que se ponen en común las necesidades de plantel de cada huerto y los insumos que se van a compartir (semillas, sustrato, alveolos); $y$ tres "hacenderas" - o jornadas de trabajo colectivo- en las que se mejoran las instalaciones y se preparan las bandejas de semillero para cada huerto, a la vez que se comparten - desde la práctica- los conocimientos sobre técnicas de semillero entre todos. El resultado del proceso es múltiple, por un lado está, naturalmente, la producción de plantel, pero tan importante como ésta es el trabajo de organización colectiva y horizontal y la solución de la problemática de su implementación entre los diferentes huertos que participan. En conjunto, los resultados de esta experiencia se vinculan con la mejora del conocimiento en la construcción de semilleros, la distribución de semillas entre los huertos y la práctica de la gestión colectiva de recursos comunes de la red y propios de cada huerto.

\section{Materia orgánica y Compostaje: Operación Estiércol y Proyecto de Seguimiento y Control del Compostaje}

Para que las semillas crezcan y den frutos es preciso un buen sustrato y la renovación constante de nutrientes, lo cual nos introduce en el segundo campo de prácticas que exponemos en este texto. En la ReHdMad!, como ocurre con todos los hortelanos agroecológicos, la renovación de nutrientes es un aspecto del trabajo que atrae una atención primordial, tanto por su importancia en una agricultura local autosostenible, como por el valor educativo de carácter ecológico que implica. Así, lógicamente, la mayoría de huertos ponen en marcha desde sus inicios la construcción de un compostador alimentado por los residuos orgánicos generados por los propios hortelanos y por otros vecinos, constituyendo a la vez una herramienta para la reducción de residuos en la ciudad y para el acercamiento a los huertos de vecinos afines. Pero la ReHdMad! da un paso y, en la línea de trabajo de investigación y profundización colectiva del conocimiento que le caracteriza, amplía el conocimiento y la formación sobre compostaje realizando talleres sobre Análisis de suelos, Vermicompostaje, Compostaje en pila y Construcción de compostadoras. Todos ellos divulgados a través de eventos con colectivos que no pertenecen a la red (Semana de la Ciencia y los Encuentros anuales) y de cursos y talleres específicos sobre estos temas.

Otras prácticas destacables en este ámbito serían, en primer lugar, la "Operación Estiércol", jornada de distribución cooperativa y coordinada de abono orgánico en los huertos que ha tenido lugar en octubre de 2012 y en noviembre de 2013. La actividad surgió al constatar la oportu-

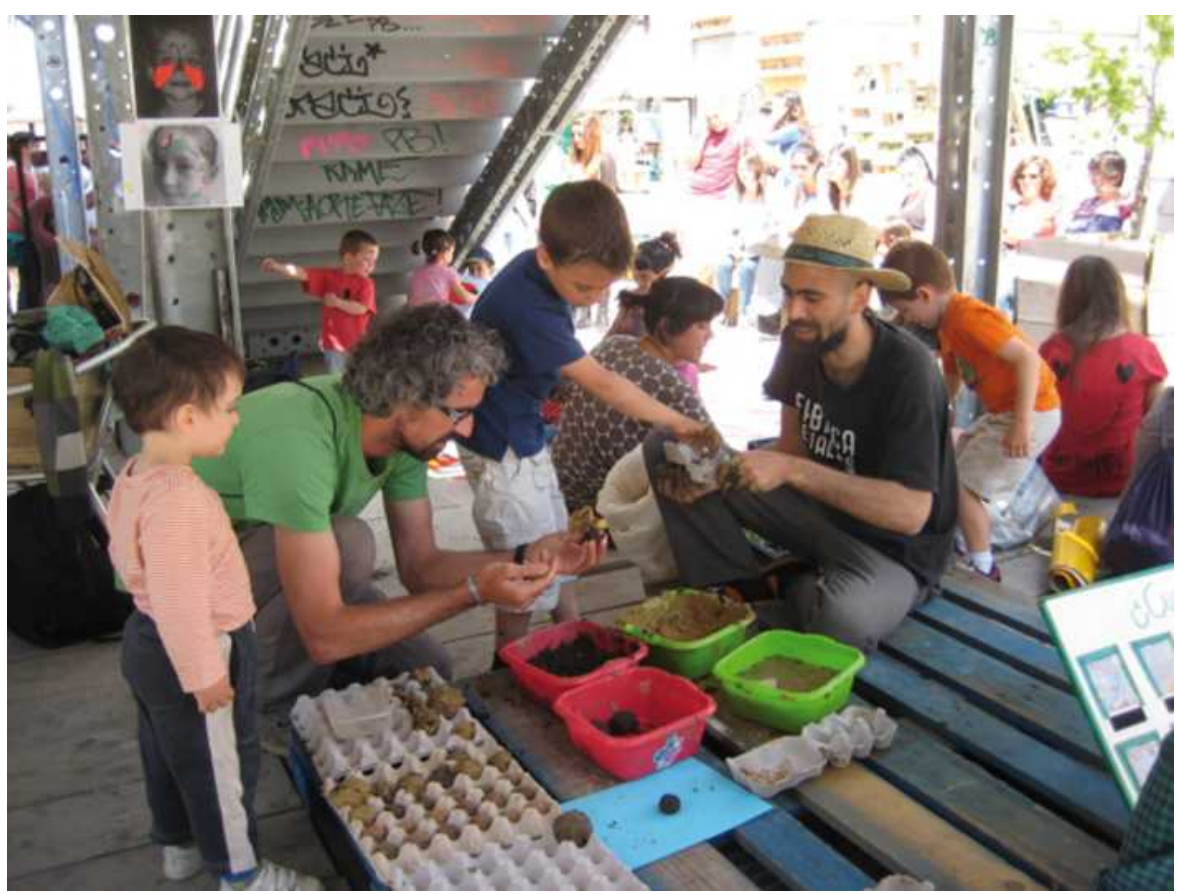


nidad de obtener gran cantidad de estiércol gratuito en la misma ciudad de Madrid, pero sin capacidad de recogerlo por no disponer de un vehículo apropiado. A continuación, gracias al trabajo en red, un hortelano comunitario, propietario de un camión basculante, lo puso a disposición del colectivo para realizar los portes. En la primera ocasión el estiércol se repartió en una jornada o "hacendera" a 11 huertos, y en la segunda se distribuyó a 18 huertos durante dos jornadas, complementando así los procesos de compostaje propios de cada huerto.

En segundo lugar mencionamos el "Proyecto de Seguimiento y Control del Compostaje de los Huertos Urbanos Comunitarios de la ReHd Mad!", que dinamiza el Huerto de Grama, con el objetivo de evaluar el compostaje colectivo en los huertos urbanos. Se analizan las formas, metodología y mantenimiento del compostaje; número y tipos de compostadores; número y tipología de personas participantes; cantidad de compost obtenido y residuos tratados, etc.; y con ello se reconstruyen herramientas para mejorar las metodologías implementadas. ${ }^{6}$ La información se recoge a través de un primer cuestionario online y de visitas concertadas a los propios huertos, donde se rellena un segundo cuestionario técnico, un fichero fotográfico y un control del compostador, con recogida de muestras. Esta información se procesa y evalúa, elaborando una ficha-resumen de cada huerto, para finalmente publicar un informe original en su presentación y global en cuanto a los aspectos estudiados.

En definitiva, el horizonte global del trabajo que guía este conjunto de prácticas se sitúa, por un lado, en un análisis de la importancia de los huertos urbanos comunitarios en cuanto a la recogida de residuos; y por otro, en su función educativa sobre el funcionamiento de los ecosistemas y la intervención de la sociedad en la sostenibilidad o, utilizando un término más lleno de contenido y sentido ecológico, en la regeneración de las ciudades y en la suya propia. Por tanto, su mensaje no es únicamente de carácter instrumental, sino formativo respecto a valores y prácticas ecológicas.

\section{Herramientas y Bioconstrucción: Construcción con Palés e "Inventacos"}

Como indicábamos al comienzo de este artículo, una de las motivaciones por las que surge la ReHdMad! es la de fomentar y reforzar la autogestión, el intercambio y las relaciones socioeconómicas no mercantiles. Con estas premisas y dada la precariedad material con la que nacen en general los huertos urbanos, el intercambio de herramientas y de información sobre dónde y cómo conseguirlas ha sido uno de los elementos sobre los cuales se ha materializado el trabajo en red. En este sentido, en el I Encuentro se identificaron ya algunas de las experiencias realizadas, como el préstamo por parte de Centros de Educación Ambiental, que se han ido consolidando y ampliando el acceso a nuevos huertos y herramientas (motoazadas, desbrozadoras, trituradoras, etc.). Igualmente, entre los componentes de la ReHdMad! se han desarrollado distintas experiencias de intercambio, tanto de materiales como de información y formación: herramientas compartidas para el Curso de Cultivo Biointensivo (palas, bieldos, hoces), el camión para

6 Para mayor información sobre este trabajo, véase http://compostajegrama. blogspot.com.es/ la "operación estiércol" e instrumentos diversos para el Taller de Construcción con Cañas.

Otra de las líneas desarrolladas en esta área consiste en la autocons- 
trucción, sustentada casi siempre en la reutilización de materiales dedicados a otros usos y recuperados para utilizarlos de forma creativa dentro de los huertos. De este modo se preparó el Taller de Construcción con Palés, en el que se compartieron conocimientos sobre su uso de para armar artefactos útiles a los huertos (semilleros, composteras, mesas de cultivo, mesas en general, bancos, etc.). De forma similar, en el II Encuentro se realizó un taller que denominamos "Inventacos" para compartir conocimientos sobre el uso de sistemas y mecanismos como hornos solares, jardineras de materia-

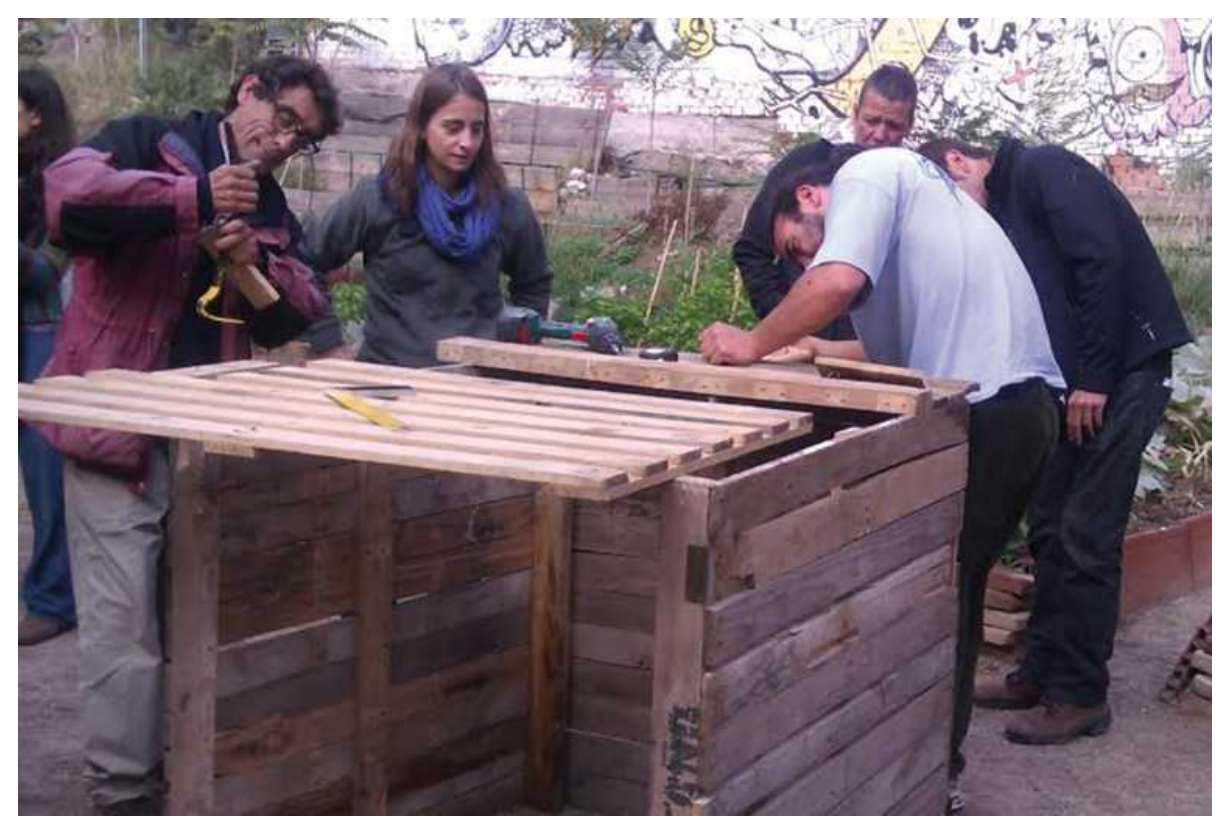
les reciclados y bicimáquinas. Y respecto a la bioconstrucción, se han impartido numerosos talleres en diferentes huertos y se han elaborado planos para la composición de aparatos concretos que hemos publicado en el blog. ${ }^{7}$

En todo caso, lo que ponen de manifiesto este conjunto de prácticas -al que cabría añadir otras como el montaje de una criba de compost con palés de gran tamaño o el ensamblaje de cubos vacíos de pintura para crear un vermicompostador de lombrices rojas-, es la capacidad de innovación y creatividad práctica de la ReHdMad!, generalmente a partir de la recuperación y reutilización de materiales desechados, apoyando así uno de los pilares de la soberanía alimentaria, que es el desarrollo y uso de "tecnologías apropiadas" para la producción de alimentos. Creatividad que se plasma también en la Exposición ambulante de fotografías y frases titulada "Vamos de Huertos", en los contenidos del blog de la red y en diferentes artilugios que inventa cada huerto para solucionar problemas concretos. Y - no debemos olvidarlo, pues es un carácter esencial de la ReHdMad! - todo ello emerge como producto de un proceso colectivo de conocimiento, intuición y emociones compartidas y disfrutadas, que añade sentido social al "trabajo".

\section{Formación y Tecnologías: Curso de Método de Cultivo Biointensivo de Alimentos}

Como es fácil deducir de lo expuesto anteriormente, todas las prácticas descritas tienen un componente formativo implícito. Constituyen en sí mismas un espacio de aprendizaje colectivo y complejo, una escuela de democracia participativa, en la que no solo se aprenden - desde la práctica y con el ejemplo- tecnologías hortícolas, sino también valores y técnicas de trabajo cooperativo, de gestión de bienes comunes y de generación de cultura ciudadana. Pero, incluso de forma explícita, en los dos Encuentros realizados y en varias asambleas se han puesto en común espacios de formación organizados por la ReHdMad! y se han recogido inquietudes de formación de sus miembros. Como consecuencia de esta estrategia, se han abordado — generalmente a través de Talleres o Seminarios participativos- multitud de temáticas que interesan a los huertos: Conflictos interpersonales, Técnicas de injerto,

7 http://redhuertosurbanosmadrid. wordpress.com/taller-de-construccioncon-palets/.

Figura 5. Inventacos. Fuente: Elaboración propia. 


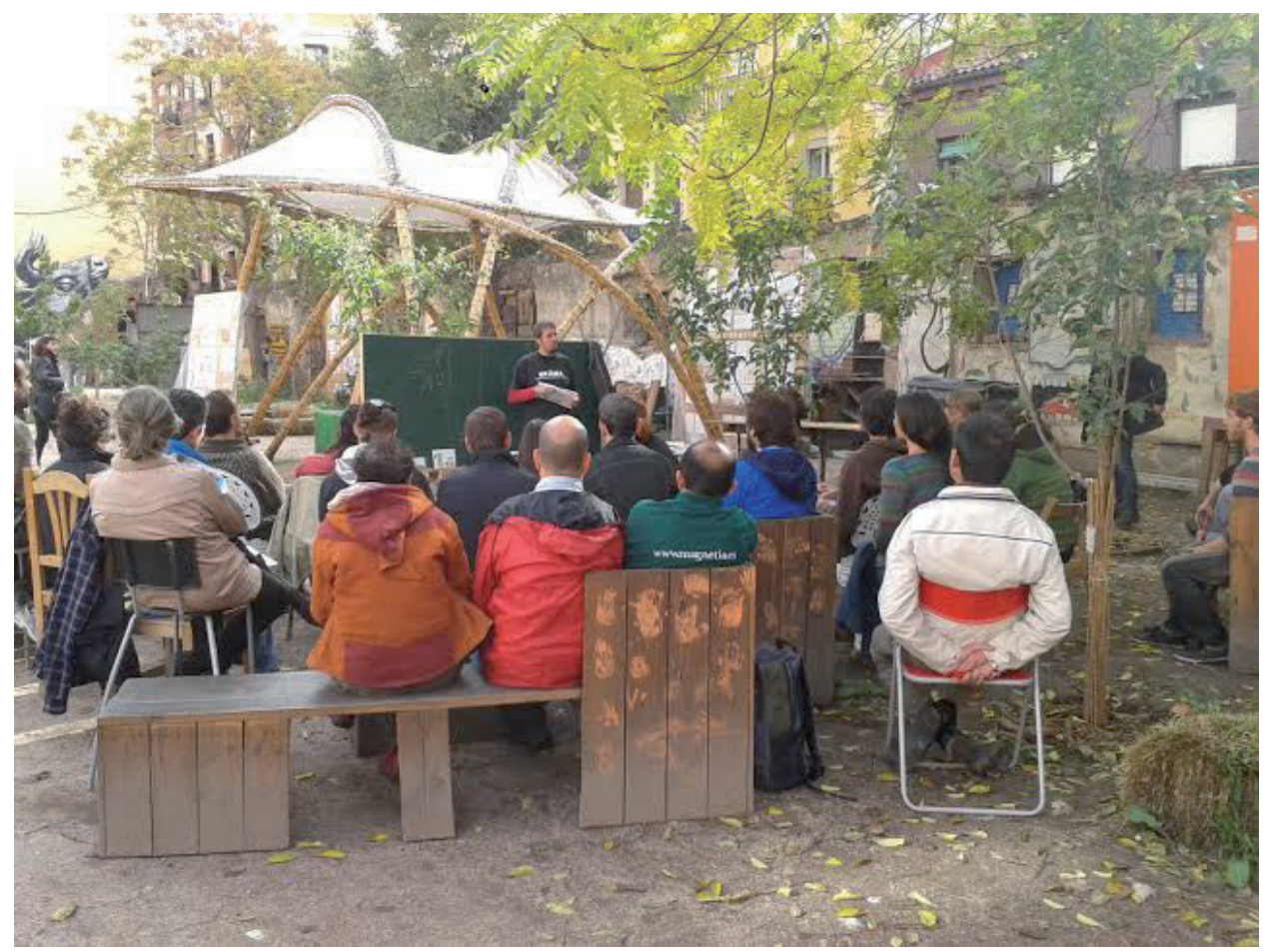

Figura 6: Aula de formación. Fuente: Elaboración propia.
8 Buena parte de los productos académicos generados se pueden consultar en la web de la HuertAula Comunitaria de Agroecología de Cantarranas: https:// portal.ucm.es./web/agroecología/trabajos-académicos.
Identificación y cultivo de plantas medicinales, Elaboración de pomada de caléndula, Prevención de riesgos en la huerta, Separación de residuos, Producción de spirulina, Jornadas universitarias sobre Ecofeminismo, etc. La filosofía de estas iniciativas formativas es coherente con la de los propios huertos de la ReHdMad!: abiertas, gratuitas, participativas, lúdicas y ecológicas. La gratuidad se logra seleccionando a los formadores/dinamizadores entre personas de los propios huertos o de otras redes de movimientos sociales con los que se comparten apoyos mutuos, estimulando así los procesos de autogestión, empoderamiento y educación colectiva transformadora.

Entre los ejemplos de este tipo de actividades formativas cabe citar los talleres sobre Recursos Pedagógicos, Recursos en Red y Escenarios de Futuro, Talleres Infantiles, Saberes Populares, y 8 Actividades oficiales en la Semana de la Ciencia de Madrid, repartidas entre los años 2011, 2012 y 2013. Los contenidos y formatos de estas 8 actividades han sido diversos, por ejemplo, jornadas de puertas abiertas y visitas guiadas; Mesa Redonda sobre "Arquitectura, urbanismo y huertos comunitarios"; "¿Qué comemos? Experiencia desde los huertos agroecológicos y comunitarios"; y bicicletada interhuertos. Por otro lado, como se indicó más arriba, se promueve la generación de conocimiento académico en áreas de Agroecología, Soberanía Alimentaria, Inclusión Social, Metodologías participativas, Ecología y Sostenibilidad, Urbanismo y Espacios Públicos ${ }^{8}$ participando activamente y/o acompañando a investigadores en el desarrollo de sus trabajos. Un ejemplo concreto de experiencia formativa lo ofrece el "Curso Teórico-práctico de Introducción al Método Biointensivo de Cultivo de Alimentos", que se organizó en el marco de las actividades de la Semana de Lucha Campesina 2013 en Madrid. La asistencia desbordó todas las previsiones, contando con 75 personas que, durante 2 días, participaron en las exposiciones teóricas y en el arduo trabajo práctico de las técnicas de doble cava o de compostaje en pila.

\section{Autonomía y regularización}

No podemos finalizar esta exposición sin citar uno de los temas y experiencias de trabajo que afectan de manera fundamental a los huertos que conforman la red. Nos referimos al hecho de que la mayoría son todavía recientes y no cesan de producirse nuevas incorporaciones, de modo que la situación legal de los huertos comunitarios es muy diversa y, en muchos casos, alegal. A este respecto, por un lado, con el fin de facilitar el avance y consolidación de las iniciativas, se ha elaborado un protocolo de creación de huertos que se encuentra accesi- 
ble en el blog de la ReHdMad! ${ }^{9}$ Por otro lado, la singularidad de estos espacios ha generado en ocasiones conflictos con la administración (multas, cortes de agua, e incluso desmantelamiento salvaje de algunos huertos), habiendo sido clave la actuación de la RedHMad! para solucionar o paliar estos graves problemas.

En ese marco, se impulsan relaciones con la Administración con objeto de facilitar el conocimiento institucional de estas experiencias y de evidenciar nuestra capacidad de interlocución. Los trabajos académicos, las publicaciones, el apoyo de la Federación de Asociaciones de Vecinos de Madrid (FRA-

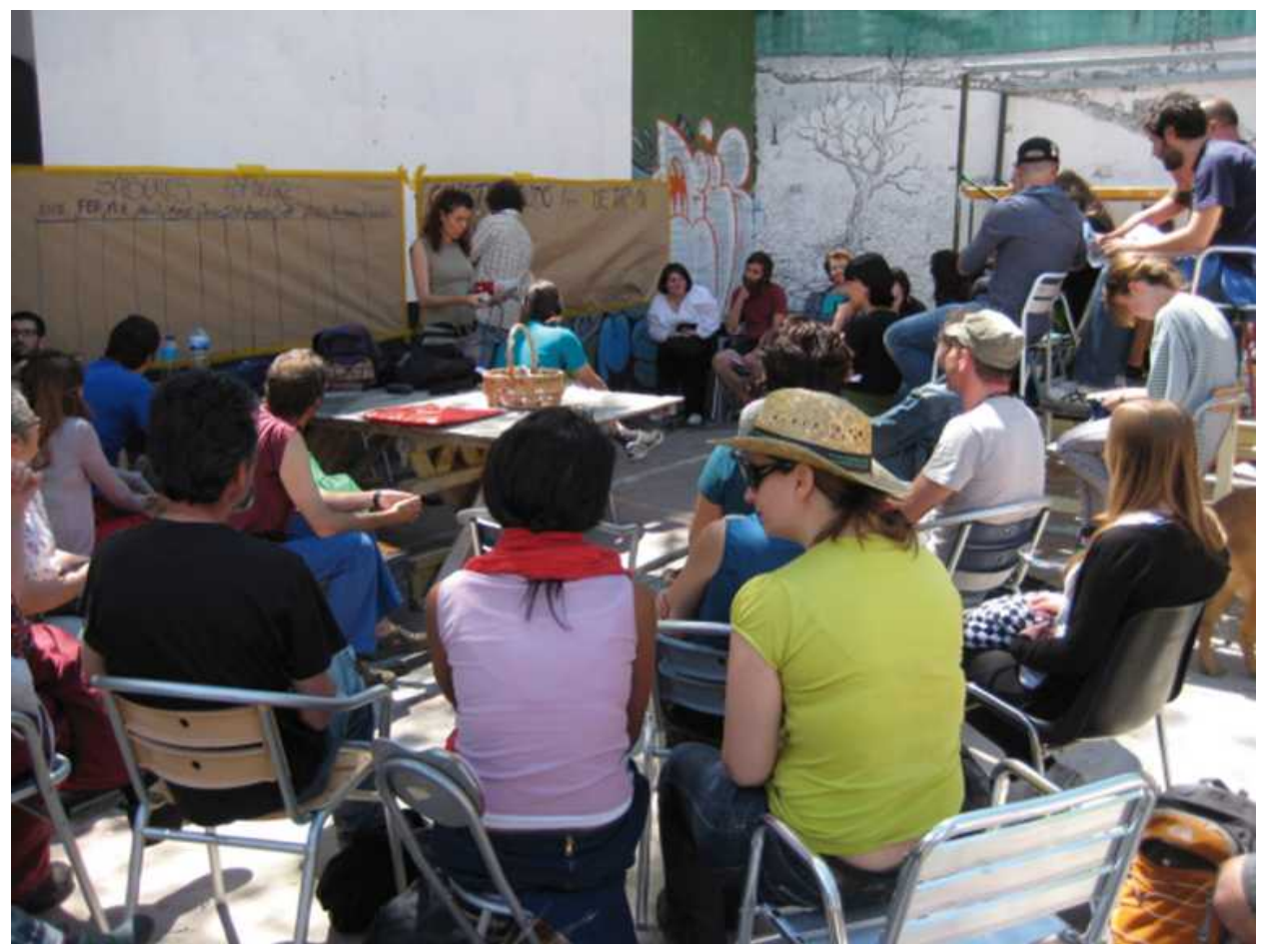
$\mathrm{VM})$ y de los vecinos en general, la visibilidad ganada a través de los medios de comunicación o el reconocimiento internacional del trabajo de la red al ser distinguida como una de las 100 mejores prácticas mundiales de sostenibilidad urbana de 2012 por el Programa Hábitat de la ONU, son algunos de los argumentos de peso que apoyan el movimiento de horticultura urbana comunitaria. Con ese bagaje y el enorme interés inherente a los propios huertos urbanos comunitarios, en el último trimestre de 2013 se están realizando reuniones con el Ayuntamiento de Madrid para regularizar los huertos existentes y reconocer el movimiento social que lo impulsa. Obviamente, este proceso no está exento de debates internos y de pactos sobre un tema básico de los huertos comunitarios, cual es la autogestión y la práctica de la agroecología en los espacios públicos, así como la aceptación de la multidimensionalidad de estas experiencias (que incluyen, por ejemplo, arte, urbanismo y uso de los espacios públicos) que superan la vertiente exclusivamente agroecológica de los huertos. El debate y las negociaciones, llevadas a cabo conjuntamente con la FRAVM, se halla en pleno desarrollo en el momento de finalizar la escritura de este texto. Esperamos que acaben de forma satisfactoria para todos.

MARTÍNEZ MADRID, Belén et al. Red de Huertos Urbanos Comunitarios de Madrid. Prácticas y reflexiones colectivas. $H a^{-}$ bitat y Sociedad, 2013, n 6, p. 129-137.

$<$ www.habitatysociedad.us.es>

http://dx.doi.org/10.12795/HabitatySociedad.2013.i6.08 
